Revista Brasileira de Agricultura Irrigada v.14, nº.3, p. 4086 - 4096, 2020

ISSN 1982-7679 (On-line)

Fortaleza, CE, INOVAGRI - http://www.inovagri.org.br

DOI: $10.7127 /$ rbai.v14n101168

Protocolo 1168.20 - 09/06/2020 Aprovado em 22/10/2020

\title{
CRESCIMENTO INICIAL DE MINIMELANCIA CV. SUGAR BABY IRRIGADA COM ÁGUAS SALOBRAS
}

\author{
Laís Monique Gomes do Ó ${ }^{1}$, Alide Mitsue Watanabe Cova², Neilon Duarte da Silva ${ }^{3}$, Petterson \\ Costa Conceição Silva ${ }^{4}$, Hans Raj Gheyi ${ }^{5}$, André Dias de Azevedo Neto ${ }^{6}$.
}

\begin{abstract}
RESUMO
Esse estudo teve como objetivo avaliar o efeito de diferentes níveis salinos da água de irrigação no crescimento inicial de minimelancia cv. Sugar Baby através de técnicas estatísticas univariada e multivariada. $\mathrm{O}$ experimento foi desenvolvido em casa de vegetação na Universidade Federal do Recôncavo da Bahia, em Cruz das Almas, BA. As sementes de minimelancia da cv. Sugar Baby foram semeadas em bandejas plásticas de polietileno contendo substrato composto de fibra de coco e húmus na proporção de 3:1 (v/v). O delineamento utilizado foi o inteiramente casualizado com quatro condutividades elétricas da água $(\mathrm{CEa}): 0,33 ; 1,5 ; 3,5 ; 5,5 \mathrm{dS} \mathrm{m}^{-1}$, com cinco repetições. As variáveis analisadas foram: tempo de emergência, índice de velocidade de emergência, teor de água, altura das plantas, número de folhas, massa fresca e seca da parte aérea. $\mathrm{O}$ aumento dos teores de sais na água de irrigação aumenta o tempo de emergência de mudas de minimelancia A altura e massa seca das plantas foram influenciadas negativamente com o aumento gradual dos níveis salinos. A CEa de até 3,5 dS m ${ }^{1}$ não interfere no crescimento inicial de minimelancia. A análise de componentes principais permite recuperar todas as informações referentes ao efeito da salinidade nas variáveis permitindo confirmar os efeitos significativos propostos pela análise univariada. A análise multivariada permite obter de forma objetiva um melhor detalhamento dos resultados. As CEa acima de 3,5 $\mathrm{dS} \mathrm{m}{ }^{-1}$ não são recomendas para a produção de mudas de minimelancia cv. Sugar Baby.
\end{abstract}

Palavras-chave: Citrullus lanatus, ambiente protegido, salinidade, estresse abiótico.

\footnotetext{
${ }^{1}$ Agrônoma, Doutoranda em Engenharia Agrícola da Universidade Federal do Recôncavo da Bahia, Rua Rui Barbosa, s/n, Cruz das Almas, Bahia, Brasil, E-mail: laisnique@ hotmail.com.

2 Agrônoma, Pós-Doutoranda no Programa de Pós-graduação de Engenharia Agrícola da Universidade Federal do Recôncavo da Bahia, Rua Rui Barbosa, s/n, Cruz das Almas, Bahia, Brasil, E-mail: alidewatanabe@yahoo.com.br.

${ }^{3}$ Agrônomo, Doutorando em Engenharia Agrícola, Universidade Federal do Recôncavo da Bahia, Rua Rui Barbosa, s/n, Cruz das Almas, Bahia, Brasil, E-mail: neylon_duart@hotmail.com.

${ }^{4}$ Agrônomo, Doutor em Engenharia Agrícola, Universidade Federal do Recôncavo da Bahia, Rua Rui Barbosa, s/n, Cruz das Almas, Bahia, Brasil, E-mail: petter.ufrb@gmail.com.

${ }^{5}$ Professor Visitante, Universidade Federal do Recôncavo da Bahia, Cruz das Almas, Bahia, E-mail: hgheyi@gmail.com. ${ }^{6}$ Professor Universidade Federal do Recôncavo da Bahia, Centro de Ciências Exatas e Tecnológicas, Rua Rui Barbosa, s/n, Cruz das Almas, Bahia, Brasil, E-mail: Azevedo_neto@yahoo.com.br.
} 


\title{
INITIAL GROWTH OF MINI WATERMELON SUGAR BABY IRRIGATED WITH BRACKISH WATER
}

\begin{abstract}
This study aimed to evaluate the response of different salt levels of irrigation water in the initial growth of mini watermelon cv. Sugar Baby through univariate and multivariate statistical techniques. The experiment was carried out in a greenhouse at Federal University of Recôncavo of Bahia in Cruz das Almas, BA. The mini watermelon seeds of cv. Sugar Baby were sown in polyethylene plastic trays, using a substrate consisting of coconut fiber and humus in 3:1 (v/v) proportion. The experimental design used was completely randomized with four electrical conductivities of water (ECw): $0.33 ; 1.5$; $3.5 ; 5.5 \mathrm{dS} \mathrm{m}^{-1}$, with five repetitions. The variables analyzed were: plant height, number of leaves, fresh and dry shoot weight, emergence time, emergence speed index and water content. The increase in the levels of salts in the irrigation water increases the emergence time of seedlings of mini watermelon. The height and dry mass of the mini watermelon plants reduced with increasing salinity. $\mathrm{ECw}$ of up to $3.5 \mathrm{dS} \mathrm{m}^{-1}$ does not compromise the initial growth of mini-watermelon. The principal component analysis allows retrieving all information regarding the effect of salinity on the variables, allowing confirming the effects proposed by the univariate analysis. A multivariate analysis allows objectively obtaining a better detail of the results. Ecw above $3.5 \mathrm{dS} \mathrm{m}^{-1}$ are not recommended to produce mini watermelon cv. Sugar Baby seedlings.
\end{abstract}

Key words: Citrullus lanatus, greenhouse, salinity, abiotic stress.

\section{INTRODUÇÃO}

A produção de melancia no Brasil, em 2017, foi de 2,31 milhões de toneladas, com uma área colhida de aproximadamente 105,06 mil hectares, com destaque para a região Nordeste que produziu 663 mil toneladas, equivalente a $28 \%$ da produção nacional (IBGE, 2019).

Destacada por possuir a maior produção de melancia do Brasil, uma parte considerável do Nordeste brasileiro apresenta clima semiárido. Devido a irregularidade das chuvas, na região semiárida o uso da irrigação na agricultura é indispensável sendo necessário o uso de água de qualidade inferior para a irrigação, como por exemplo, as águas salobras ou subterrâneas (NEVES et al., 2017). Para Sousa et al. (2016) o uso de águas residuais ou de qualidade inferior na agricultura seria uma boa opção, especialmente em períodos de escassez hídrica.

Contudo, quando as sementes são submetidas à salinidade, o excesso de sais afeta a capacidade de absorção de água, que induz alterações bioquímicas e osmóticas na germinação e, consequentemente na emergência das plântulas (NÓBREGA et al., 2020) e na produção de massa seca das mudas de melancia (SILVA JÚNIOR et al., 2020; NÓBREGA et al., 2020). Embora, os efeitos podem ocorrer em todos os estádios de desenvolvimento, todavia a germinação e o crescimento inicial das plântulas são as fases mais afetadas pela salinidade, na maioria das plantas (SARABI et al., 2016).

A influência da salinidade no vigor, qualidade das sementes e acúmulo de biomassa tem sido reportadas em mudas de melancia por Silva Júnior et al. (2020) e Nóbrega et al. (2020), onde os autores observaram deterioração da qualidade de sementes e redução no acúmulo na biomassa seca de plantas. Portanto, avaliar o nível de tolerâncias das plantas aos sais é fundamental em áreas afetadas que podem comprometer a implantação da cultura (COVA et al., 2016), principalmente na fase de produção de mudas de melancia que está em ascensão científica (SILVA JÚNIOR et al., 2020).

Diante do exposto, a aplicação de técnicas multivariadas em pesquisas sob condições de estresse salino pode ajudar a definir os indicadores efetivos para a 
tolerância, aumentar a compreensão das respostas das diferentes cultivares de uma espécie (SARABI et al., 2016; AYADI et al., 2018) e contribuir para a tomada de decisões em planejamentos ambientais.

Por se tratar de uma cultura de grande importância econômica com vistas a sua inserção em regiões semiáridas, este estudo teve como objetivo avaliar o efeito de diferentes níveis salinos na germinação e crescimento inicial de minimelancia cv. Sugar Baby através de técnicas estatísticas univariada e multivariada.

\section{MATERIAL E MÉTODOS}

O experimento foi desenvolvido em casa de vegetação, pertencente a Universidade Federal do Recôncavo da Bahia, Cruz das Almas, BA, a 12 ${ }^{\circ} 40^{\prime} 19^{\prime \prime} \mathrm{S}, 39^{\circ}$ 6' 23" O, 220 $\mathrm{m}$ de altitude. Segundo a classificação de Köppen, o clima é tipo tropical quente e úmido (Af), sem estação seca, com umidade relativa e temperatura média anuais de $80 \%$ e $24{ }^{\circ} \mathrm{C}$, respectivamente, apresentando pluviosidade média anual de $1.143 \mathrm{~mm}$.

O substrato utilizado foi composto pela mistura de fibra de coco e húmus na proporção de 3:1 (v/v), o qual foi adicionado às bandejas plásticas de polietileno de 50 células. Em cada célula foi depositada uma semente de minimelancia cv. Sugar Baby a 2,0 cm de profundidade.

O delineamento estatístico utilizado foi o inteiramente casualizado com quatro tratamentos e cinco repetições, cada repetição contendo 10 células de plântulas. Os tratamentos consistiram na utilização de quatro condutividades elétricas da água de irrigação (CEa): 0,$33 ; 1,5 ; 3,5 ; 5,5 \mathrm{dS} \mathrm{m}^{-1}$. Os níveis de $\mathrm{CEa}$ foram estabelecidos considerando-se a cultura da melancieira como moderadamente sensível ao estresse salino, sendo a salinidade limiar de $2,2 \mathrm{dS} \mathrm{m}^{-1}$ no extrato de saturação do solo (correspondente a $1,5 \mathrm{dS} \mathrm{m}^{-1}$ na água) (AYERS; WESTCOT, 1999).

Para obtenção dos diferentes níveis de salinidade foi utilizada água de abastecimento local $\left(\mathrm{CEa}=0,33 \mathrm{dS} \mathrm{m}^{-1}\right)$ com adição de cloreto de sódio $(\mathrm{NaCl})$ a diferentes proporções. No preparo da água dos tratamentos foi considerada a relação entre CEa e concentração de sais, extraída de Richards (1954), conforme a Eq. 1:

$$
\mathrm{Q} \mathrm{NaCl}\left(\mathrm{mg} \mathrm{L}^{-1}\right)=640 \times \mathrm{CEa}\left(\mathrm{dS} \mathrm{m}^{-1}\right)
$$

Em que: Q é a quantidade de sal a ser dissolvido na água; e CEa é a condutividade elétrica da água desejada.

Antes da semeadura, elevou-se o teor de umidade do substrato até alcançar a capacidade de campo, utilizando água de abastecimento local. A irrigação foi realizada diariamente, aplicando-se em cada unidade experimental a reposição diária com respectivas soluções, com volume de $50 \mathrm{~mL}$ por tratamento, aplicada pela manhã e pela tarde.

Ao longo do experimento os valores de $\mathrm{pH}$ e condutividade elétrica das soluções (CEa) foram monitoradas por meio de medidores portáteis de $\mathrm{pH}$ e de condutividade com resoluções de 0,01 e 0,01 dS $\mathrm{m}^{-1}$, respectivamente, e com compensação automática de temperatura $\left(25^{\circ} \mathrm{C}\right)$.

No final do período experimental (15 DAS - dias após semeadura) foram avaliados a altura de plantas (AP), utilizando uma régua graduada; o número de folhas (NF); a massa fresca da parte aérea (MFPA) e a massa seca da parte aérea (MSPA), ambas determinadas em balança analítica $(0,0001 \mathrm{~g})$. A MSPA foi determinada após a secagem das plantas em estufa com circulação forçada de ar a $65^{\circ} \mathrm{C}$, por $72 \mathrm{~h}$. O teor de água (TA) foi determinado conforme Barros et al. (2010) utilizando a Eq. 2:

$\% \mathrm{TA}=\frac{\text { MFPA-MSPA }}{\text { MFPA }} \times 100$

Em que: TA é o teor de água, em \%; MFPA é a massa fresca parte aérea, em g; e MSPA é a massa seca parte aérea, em g.

Durante o experimento as plântulas foram monitoradas diariamente, levando-se em consideração apenas aquelas com cotilédones acima do nível do substrato, obtendo-se um valor cumulativo. 
Assim, conforme metodologia proposta por Edmond e Drapala (1958), foi obtido o tempo médio necessário para atingir a emergência máxima (dias) e o índice de velocidade de emergência (IVE) em sementes por dia mediante as Eqs. 3 e 4, respectivamente.

$\mathrm{TE}=\frac{\left(\mathrm{N}_{1} \mathrm{G}_{1}\right)+\left(\mathrm{N}_{2} \mathrm{G}_{2}\right)+\ldots \ldots .+\left(\mathrm{N}_{\mathrm{n}} \mathrm{G}_{\mathrm{n}}\right)}{\mathrm{G}_{1}+\mathrm{G}_{2}+\ldots \ldots \ldots+\mathrm{G}_{\mathrm{n}}}$

$\operatorname{IVE}=\frac{\left(\mathrm{G}_{1}\right)}{\mathrm{N}_{1}}+\frac{\left(\mathrm{G}_{2}\right)}{\mathrm{N}_{2}}+\ldots+\frac{\left(\mathrm{G}_{\mathrm{n}}\right)}{\mathrm{N}_{\mathrm{n}}}$

Em que: TE é o tempo médio necessário para atingir a emergência máxima, em dias; $\mathrm{G}$ é o número de plântulas emergidas observadas em cada contagem; e $\mathrm{N}$ é o número de dias da semeadura a cada contagem.

Os dados foram testados quanto à normalidade (teste de Shapiro-Wilk) e posteriormente submetidos à análise de variância (ANOVA) pelo teste $\mathrm{F}(\mathrm{p}<0,05)$. Quando significativo pelo teste $\mathrm{F}$, os dados foram analisados por meio de regressão polinomial. Os modelos foram escolhidos em função de sua significância pelo teste $\mathrm{F}$ e pelo coeficiente de determinação $\left(\mathrm{R}^{2}\right)$. Todas as análises foram realizadas com o auxílio do software $\mathrm{R}$ ( $\mathrm{R}$ Development Core Team, 2017), versão 3.3.4. Os dados também foram analisados por meio de Análise de Componentes Principais (ACP), feita com o mesmo software $R$, c onforme Pagès e Husson (2001) e Viana et al. (2018).

Para tanto, foi realizada a padronização das variáveis originais em um novo conjunto de dados, conforme mostra a Eq. 5.

$\mathrm{Z}=\frac{\mathrm{X}-\overline{\mathrm{X}}}{\mathrm{S}}$

Em que: Z é o valor padronizado de X;
$\overline{\mathrm{X}}$ é a média da característica; e $\mathrm{S}$ é o respectivo desvio padrão.

A análise de componentes principais a partir dos eixos $(1,2,3 \ldots$ n) permite a visualização dos resultados sob uma ótica do percentual em que o fenômeno em estudo pode ser explicado ou a forma como as informações originais podem ser recuperadas.

Assim, o primeiro componente principal (Dim 1) explica o maior percentual da variância total. O segundo componente principal (Dim 2) explica a segunda maior percentagem e assim por diante, até que toda a variância é explicada, totalizando $100 \%$.

A incerteza associada a projeção de pontos no plano, conforme sugerido por Saporta e Hatabian (1986), é dada pela Eq. 6.

$\mathrm{PC}_{\mathrm{i}}=\mathrm{e}_{\mathrm{i}}^{\mathrm{t} x}=\mathrm{e}_{\mathrm{i} 1} \mathrm{x}_{1}+\mathrm{e}_{\mathrm{i} 2} \mathrm{x}_{2}+\ldots+\mathrm{e}_{\mathrm{ip}} \mathrm{x}_{\mathrm{p}}$

Em que: $\mathrm{e}_{\mathrm{ip}}$ é o p-ésimo auto vetor; e x é o pésimo valor da variável original.

A técnica de análise de cluster é baseada na classificação de observações ao grupo de dados homogêneos em classes (clusters) com base nas semelhanças de uma classe e dissimilaridades entre diferentes classes (NADIRI et al., 2013). Esta é uma ferramenta eficaz para a identificação e seleção de amostras homogêneas grupos dos dados hidroquímicos (BELKHIRI et al., 2012).

\section{RESULTADOS E DISCUSSÃO}

Os resultados da análise de variância revelaram efeito significativo dos tratamentos para as variáveis TE, IVE, AP e MSPA $(p<0,01)$ e para as variáveis de TA e MFPA $(p<0,05)$. No entanto, não houve efeito significativo $(\mathrm{p}>0,05)$ dos níveis de salinidade da água para o NF (Tabela 1).

Tabela 1. Resumo da análise de variância para as variáveis tempo médio necessário para atingir a emergência máxima (TE) em dias, índice de velocidade de emergência (IVE) em sementes dia ${ }^{-1}$, altura 
de plantas (AP) em cm, número de folha (NF), massa fresca (MFPA) e seca da parte aérea (MSPA) em g e teor de água (TA) em \%, de minimelancia cv. Sugar Baby irrigada com diferentes condutividades elétricas da água de irrigação $(\mathrm{CEa})$.

\begin{tabular}{|c|c|c|c|c|c|c|c|c|}
\hline \multirow{2}{*}{$\begin{array}{l}\text { Fonte de } \\
\text { variação }\end{array}$} & \multirow[b]{2}{*}{ GL } & & \multicolumn{6}{|c|}{ Quadrado médio } \\
\hline & & TE & IVE & AP & NF & MFPA & MSPA & TA \\
\hline Tratamento & 3 & $0,24^{* *}$ & $45,56^{* *}$ & $171,75^{* *}$ & $0,22^{\text {ns }}$ & $50,26^{*}$ & $0,55^{* *}$ & $0,93^{*}$ \\
\hline Resíduo & 16 & 1,87 & 5,78 & 17,12 & 0,09 & 10,28 & 0,07 & 0,27 \\
\hline $\mathrm{CV} \%$ & & 19,45 & 14,76 & 13,83 & 8,92 & 20,93 & 20,05 & 9,53 \\
\hline Média Geral & & 3,21 & 0,71 & 9,46 & 3,30 & 15,32 & 1,39 & 90,88 \\
\hline
\end{tabular}

GL - Graus de liberdade; ${ }^{*},{ }^{* *}$ significativo em nível de 0,05 e 0,01 de probabilidade pelo teste $\mathrm{F} ;{ }^{\text {ns }}$ - não significativo; $\mathrm{CV}$ - Coeficiente de variação

Pela análise da Figura 1, pode-se observar que a salinidade aumentou linearmente o TE e diminuiu o IVE e a AP das plântulas de minimelancia cv. Sugar Baby.
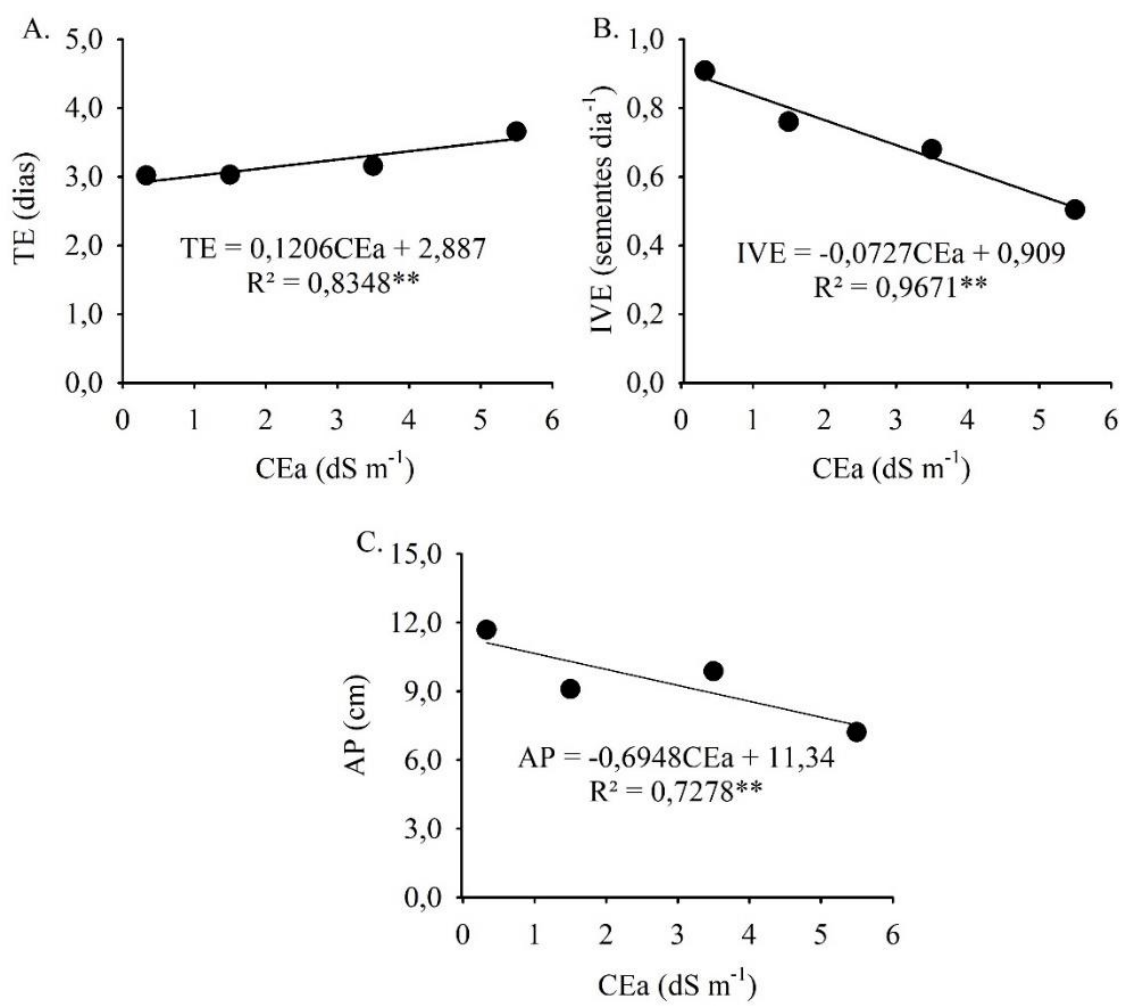

Figura 1. Tempo médio necessário para atingir a emergência máxima - TE (A), índice de velocidade de emergência - IVE (B) e altura de planta - AP (C) de minimelancia cv. Sugar Baby em função da condutividade elétrica da água de irrigação (CEa).

O tempo médio de emergência das plântulas de minimelancia apresentou acréscimos de 0,1206 dias em função do aumento unitário da salinidade da água (Figura 1A). Dessa forma, quando as plântulas foram irrigadas com o maior nível de salinidade $(5,5$ $\mathrm{dS} \mathrm{m}^{-1}$ ) houve um aumento de $21,3 \%$ no tempo de emergência em relação às plantas irrigadas com o menor nível de salinidade $\left(0,33 \mathrm{dS} \mathrm{m}^{-1}\right)$. Resultados contraditórios foram observados por Silva Júnior et al. (2020) ao avaliarem o tempo de emergência de melancia cv. 'Crimson Swee' irrigada com água de salinidade 2,5 dS $\mathrm{m}^{-1}$ cultivada a pleno sol. Para esses autores o tempo de emergência variou conforme o substrato de 5 a 8 dias.

Os resultados do presente estudo podem ser explicados pelo fato de que o aumento da concentração de sais reduz a absorção de água podendo ocasionar atraso na emergência de plântulas. Nesse sentido, Nóbrega et al. (2020) enfatizam que o excesso de sais no substrato, 


\section{do Ó et al.}

proveniente da água de irrigação, reduz o potencial osmótico, diminuindo a absorção de água e causando alterações bioquímicas na germinação das sementes.

Em relação ao IVE e AP observa-se que a salinidade da água de irrigação promoveu redução linear de 7,9 e 6,1\%, respectivamente, por incremento unitário da condutividade elétrica da água de irrigação (CEa) (Figura 1B e 1C). Ao relacionar o menor e maior tratamento de salinidade, podemos verificar uma redução de $42,5 \%$ no IVE e de $32,3 \%$ na AP de minimelancia cv. Sugar Baby. Resultados semelhantes foram observados por Nóbrega et al. (2020) que o IVE e o comprimento de plântula diminuíram com o aumento da condutividade elétrica da água de irrigação.

Dentre os efeitos mais comuns da

salinidade, estão a restrição do crescimento e desenvolvimento das plantas. Rodrigues et al. (2018) enfatizam que o estresse salino causa
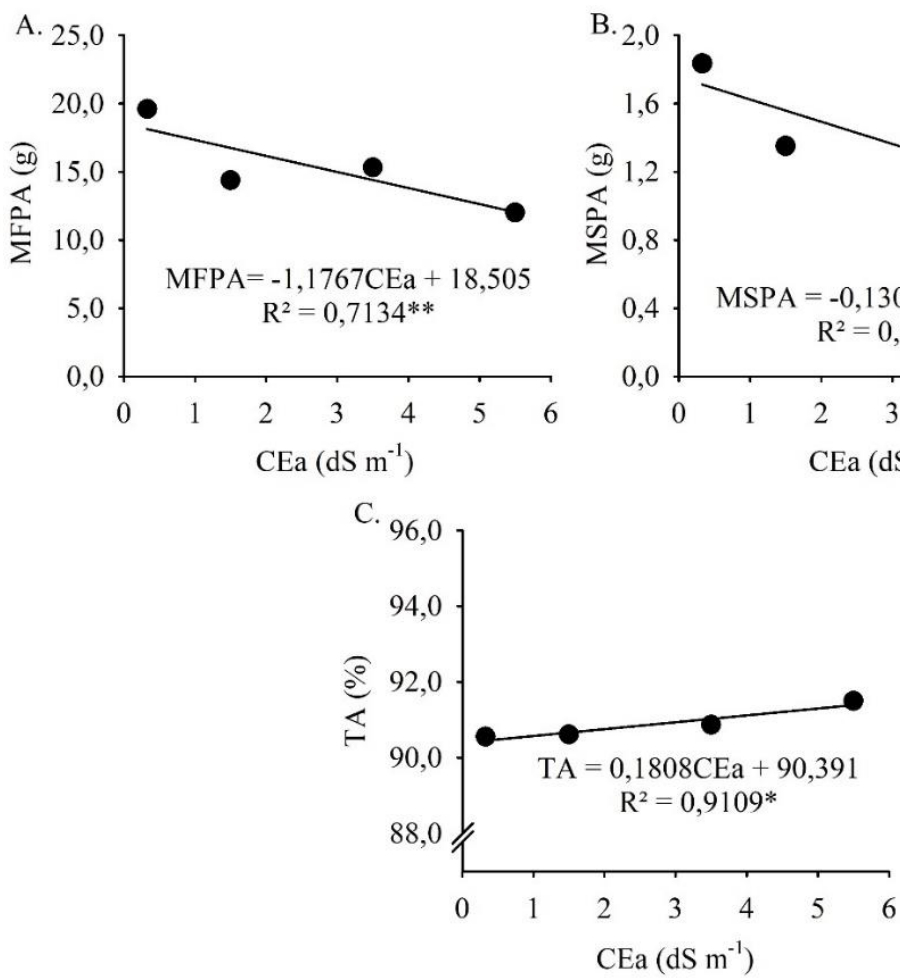

Figura 2. Massa fresca - MFPA (A) e massa seca-MSPA (B) e teor de água (C) da parte aérea das plantas de minimelancia cv. Sugar Baby em função da condutividade elétrica da água de irrigação (CEa).

Uma menor produção de matéria fresca e seca nos níveis de salinidade mais elevados podem também estar associada à redução do potencial osmótico do substrato, limitando a

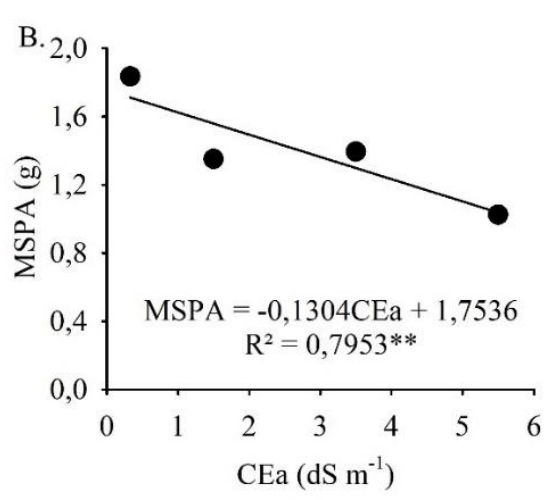

redução na turgência devido à diminuição do conteúdo de água, reduzindo a expansão celular $\mathrm{e}$, consequentemente, o crescimento das plantas.

No que se refere ao efeito dos tratamentos na variável NF não foi observada influência significativa pelo estresse salino, apresentando valores médios de 3,3 folhas por plantas (Tabela 1). Semelhante ao resultado deste estudo, Dias et al. (2015) não encontraram diferença significativa para NF até 25 dias após semeadura na cultura do melão.

A MFPA foi afetada significativamente pelo acréscimo dos níveis de salinidade na água de irrigação (Figura 2A) com decréscimo de $1,1767 \mathrm{~g}(6,3 \%)$ para cada incremento unitário da CEa, observando-se, portanto, redução de $33,6 \%$ sob maior nível de salinidade $\left(5,5 \mathrm{dS} \mathrm{m}^{-}\right.$ ${ }^{1}$ ) em relação às plantas controle (sem adição de $\mathrm{NaCl}$ ).

O mesmo ocorreu para a variável MSPA (Figura 2B), com redução de 39,4\%. 
A redução no acúmulo de massa fresca e seca sob salinidade tem sido constatada por Nóbrega et al. (2020) onde os autores atribuíram as diminuições na MS a alterações que a salinidade promove nos processos fisiológicos e bioquímicos das plantas.

$\mathrm{Na}$ Figura 2C observa-se um aumento no teor de água na plântula com a salinidade. As plantas quando submetidas a condições adversas como estresse salino alteram suas características morfológicas (DINIZ et al., 2018), assim acumulam água, tanto para

A

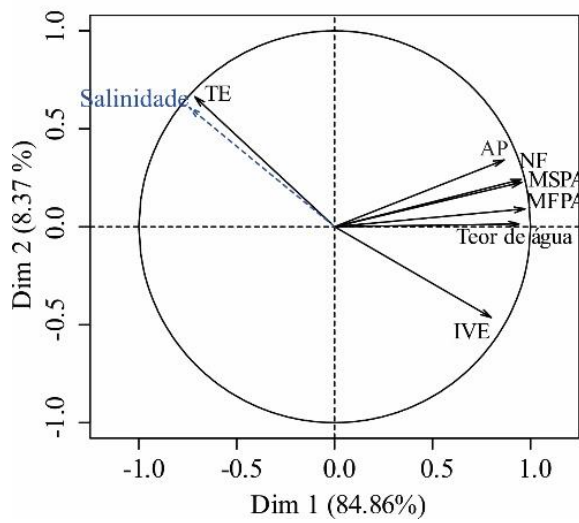

armazenamento como para diluição dos íons tóxicos $\mathrm{Na}^{+}$e $\mathrm{Cl}^{-}$(COVA et al., 2016). No entanto, neste estudo o aumento foi mínimo $(0,20 \%)$ por incremento unitário da salinidade).

$\mathrm{Na}$ Figura 3A representa a análise de componentes principais na qual, as variáveis são apresentadas no espaço e através de setas associadas a uma direção, indicando um nível de importância da variável em explicar o fenômeno (efeito da salinidade).

B

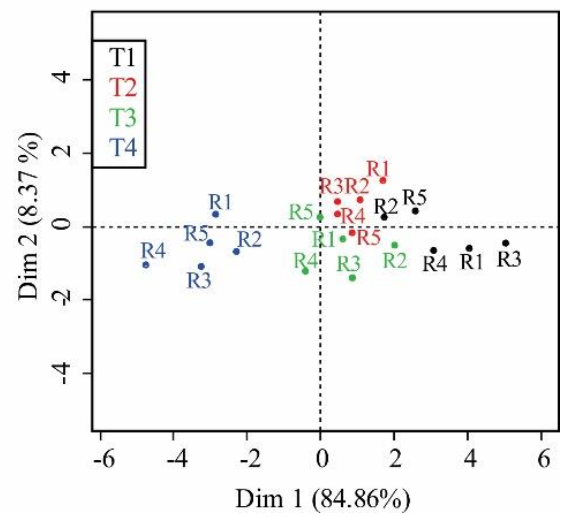

Figura 3. Análise de componentes principais (A) e agrupamento das variáveis (B) do crescimento inicial de minimelancia cv. Sugar Baby sob diferentes condutividades elétricas da água de irrigação (T1 - 0,33; T2 - 1,5; T3 - 3,5; T4 - 5,5 dS m $\left.{ }^{1}\right)$.

$\mathrm{O}$ vetor salinidade está no sentido oposto às variáveis analisadas (AP, MFPA, NF, MSPA e IVE). Em outras palavras, o fator salinidade é inversamente proporcional ao efeito causado no crescimento das plântulas. Isso implica dizer que os altos níveis de sais presentes na água reduzem as variáveis AP, NF, MFPA, MSPA e IVE.

Sousa et al. (2016) observaram que o aumento da salinidade diminuiu as variáveis de crescimento de minimelancia cv. Smile. A análise univariada através das regressões, indica que o aumento nos níveis de salinidade promoveu um acréscimo do TE. Isso foi confirmado pela análise multivariada, em que os vetores dessas variáveis estão no mesmo sentido, ou seja, apresentam correlação positiva direta.

A variável TA por apresentar baixa variação em termos de valores, possui comportamento atípico, quando comparado com as demais variáveis. Para tanto, o vetor TA na Figura 3A possui valor zero no que diz respeito ao eixo Dim2, eixo este que possui menor percentual $(8,37 \%)$ de explicação das variáveis.

Uma germinação rápida é uma característica de grande importância para alcançar altas produtividades. Sendo o TE uma variável afetada pela salinidade, essa correlação positiva é uma ferramenta importante na definição da salinidade que se deve usar na fase de emergência de plântulas.

Os dois primeiros eixos (Dim 1 e Dim 2) na ACP resumem bem o efeito dos níveis de sais no crescimento inicial da minimelancia. $\mathrm{O}$ primeiro componente resume as informações contidas na tabela de dados incluindo as repetições, concentrando a maioria das informações, enquanto o segundo fornece uma porcentagem menor, mas complementar. A soma das porcentagens de explicação dos 


\section{do Ó et al.}

componentes permite deduzir a taxa de perda de informação dos dados. Os eixos apresentam um percentual de inércia de 8,37 e $84,86 \%$, obtendo um percentual acumulado de $93,23 \%$.

A Figura 3B, apresenta a dispersão de cada repetição dentro dos eixos (Dim 1 e Dim 2). Separados por cores, verifica-se a formação de dois grupos, um contendo os tratamentos 1 , 2 e 3, e mais afastado, o tratamento 4. Dessa forma, verifica-se que a salinidade de água de até $3,5 \mathrm{dS} \mathrm{m}^{-1}$ (T3) para o crescimento inicial de minimelancia, é mais homogêneo, pois os indivíduos analisados estão menos dispersos quando comparados aos indivíduos submetidos aos tratamentos 1 e 2 (T1 e T2). O grande grupo formado pelos tratamentos 1,2 e 3 , indica um efeito semelhante nas variáveis de crescimento inicial de plântulas.

A análise de componentes principais tem

sido bastante utilizada como ferramenta auxiliar na análise de crescimento de plantas. Uma das vantagens é uma análise simultânea de diferentes variáveis para elevar a precisão dos dados da planta em diferentes níveis de sanidade (AYADI et al., 2018). A exemplo disso, Dezfuli et al. (2016), quando em teste de germinação com variedades de alfafa submetidas a diferentes níveis de salinidade verificaram uma correlação positiva entre o peso seco da parte aérea, comprimento da radícula, comprimento da plântula em função dos níveis de sais aplicados. No primeiro eixo da ACP, o Dim 1 segundo os autores pode ser representativo indicando um índice potencial de desempenho e componente de tolerância à salinidade.

Conforme confirmado por Sarabi et al. (2016), a análise de componentes principais (ACP) pode ser usada como um indicador preciso e fácil para verificar a influência da salinidade na germinação de melão.

O agrupamento hierárquico dos indivíduos analisados em cada nível de salinidade é mostrado na Figura 4.

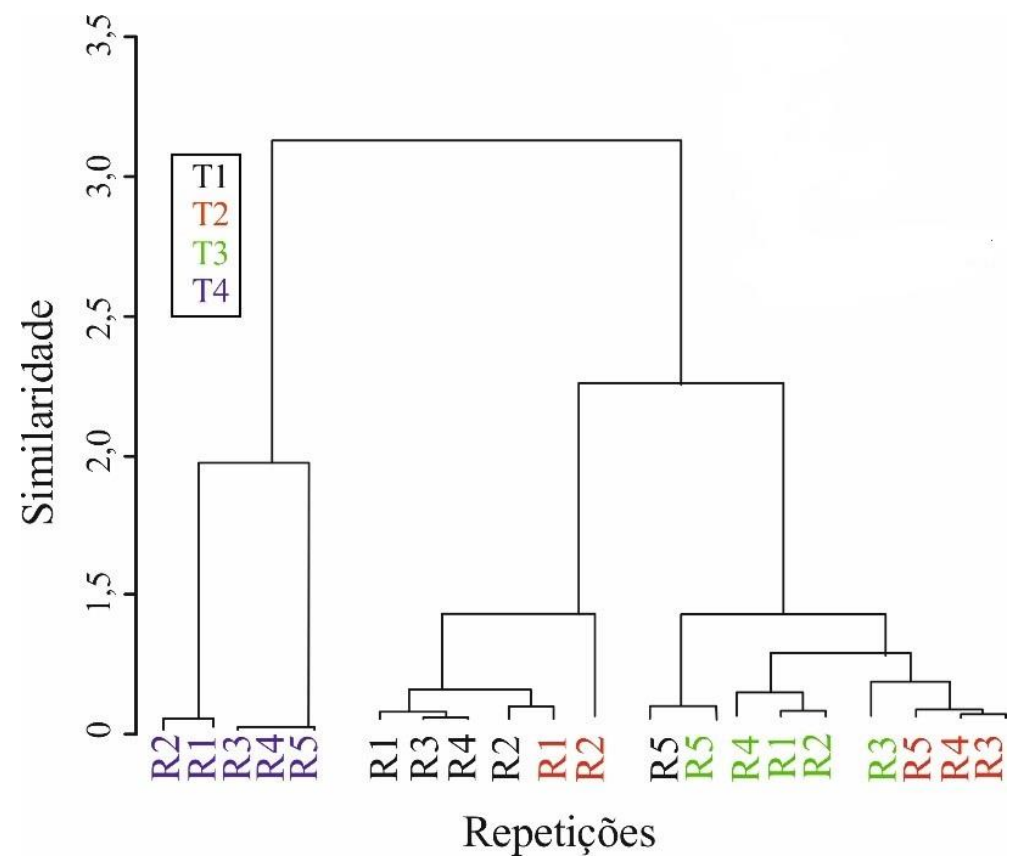

Figura 4. Agrupamento hierárquico das variáveis do crescimento inicial de minimelancia cv. Sugar Baby irrigadas com águas de diferentes condutividades elétricas da água (CEa). Legenda: (T1 - 0,33; T2 - 1,5; T3 - 3,5; T4 - 5,5 dS m²).

O dendograma (Figura 4) permite inferir que os tratamentos 0,$33 ; 1,5$ e $3,5 \mathrm{dS} \mathrm{m}^{-1}$ fazem parte do mesmo grupo, conforme apresentado na Figura 3B. Isso significa que água com salinidade de até $3,5 \mathrm{dS} \mathrm{m}^{-1}$ não afeta $\mathrm{o}$ crescimento inicial da minimelancia. Em contrapartida, valores na ordem de $5,5 \mathrm{dS} \mathrm{m}^{-1}$ se tornam prejudiciais na formação das mudas. Portanto, para a produção de mudas de minimelancia não é Sugar Baby, não é recomendada a irrigação com água de salinidade superior a $3,5 \mathrm{dS} \mathrm{m}^{-1}$. 
O dendograma na Figura 4 indica que os dois primeiros eixos da ACP são suficientes para analisar os dados. As informações de similaridade entre os indivíduos podem ser recuperadas através dos eixos Dim 1 e Dim 2. A análise dos indivíduos a partir do terceiro e quarto eixo (Dim 3 e Dim 4) não teria informações que representassem o efeito da salinidade na germinação da minimelancia.

\section{CONCLUSÕES}

A germinação, índice de velocidade de emergência, altura e massa seca das plantas de minimelancia cv. Sugar Baby foram influenciadas negativamente com o aumento gradual dos níveis salinos, não sendo recomendado para produção de mudas a utilização de águas para irrigação com salinidade superior à $3,5 \mathrm{dS} \mathrm{m}^{-1}$.

A análise multivariada cujos eixos expressam o grau de representatividade dos resultados permite uma análise mais robusta dos resultados, quando comparado com uma análise univariada (teste F), saindo das análises quantitativas feitas a partir de regressões.

\section{AGRADECIMENTOS}

Os autores agradecem à Coordenação de Aperfeiçoamento de Pessoal de Nível Superior (CAPES) pela concessão de bolsa de estudo a primeira autora, ao Conselho Nacional de Desenvolvimento Científico e Tecnológico (CNPq), a Fundação de Amparo à Pesquisa do Estado da Bahia (FAPESB) e à Universidade Federal do Recôncavo da Bahia (UFRB) pelo apoio financeiro.

\section{REFERÊNCIAS BIBLIOGRÁFICAS}

ARAÚJO, E. B. G.; SÁ, F. V. S.; OLIVEIRA, F. A.; SOUTO, L. S.; PAIVA, E. P.; SILVA, M. K. N.; MESQUITA, E. F.; BRITO, M. E. B. Crescimento inicial e tolerância de cultivares de meloeiro à salinidade da água. Revista
Ambiente \& Água, v. 11, p. 462-471, 2016. DOI: http://dx.doi.org/10.4136/1980-99.

AYADI, R.; AYED, R. B.; LASSOUED, N.; SALEM, H. B.; KHOUJA, M. L.; HAMROUNI, L.; HANANA, M. Multivariate statistical analyses for studying kenaf germination, growth, and fiber production under salinity constraint. Turkish Journal of Agriculture and Forestry, v. 42, p. 322-333, 2018. DOI: http://dx.doi.org/10.3906/tar180116.

AYERS, R. S.; WESTCOT, D. W. A qualidade da água na agricultura. 2.ed. Campina Grande: UFPB, 1999. 153p. FAO. Estudos de Irrigação e Drenagem, 29 revisado.

BARROS, H. M. M.; GHEYI, H. R.; LOGES, V.; SANTOS, M. da S.; SOARES, F. A. L. Influência da salinidade da água no crescimento de seis genótipos de helicônias.

Revista Brasileira de Horticultura Ornamental, v. 16, p. 139-145, 2010. DOI: http://dx.doi.org/10.14295/rbho.v16i2.554.

BELKHIRI, L.; MOUNI, L.; TIRI, A. Waterrock interaction and geochemistry of groundwater from the Ain Azel aquifer, Algeria. Environmental Geochemistry and Health, v. 34, p. 1-13, 2012.

COVA, A. M. W; AZEVEDO NETO, A. D. de; RIBAS, R. F; GHEYI, H. R.; MENEZES, R. V. Inorganic solute accumulation in noni (Morinda citrifolia Linn) under salt stress during initial growth. African Journal of

Agricultural Research, v. 1, p. 3347-3354, 2016. DOI: http://dx.doi.org/10.5897/AJAR2016.11416.

DEZFULI, A. A. S.; DEHCHESHMEH, S. M.; BOROUJENI, F. R.; SHIRAN, B. Evaluation of salinity tolerance of alfalfa genotypes during germination stage using multivariateanalysis. Journal of Applied Environmental and Biological Sciences, v. 6, p. 51-56, 2016. 
DIAS, N. da S.; PALÁCIO, V. S.; MOURA, K. K. C. de F.; SOUSA NETO, O. N. Crescimento do meloeiro em substrato de fibra de coco com solução nutritiva salina. Irriga, v. 20, p. 1-12, 2015. DOI: http://dx.doi.org/10.15809/irriga.2015v20n1p01.

DINIZ, G. L.; SALES, G. N.; SOUSA, V. F. de O.; ANDRADE, F. H. A. de; SILVA, S. S. de; NOBRE, R. G. Papaya production under water salinity irrigation and phosphate fertilization. Revista de Ciências Agrárias, v. 41, p. 231240, 2018. DOI: http://dx.doi.org/10.19084/RCA17067.

EDMOND, J. B.; DRAPALA, W. J. The effects of temperature, sand and soil, and acetone on germination of okra seeds. Proceedings of American Society Horticultural Science, v. 71, p. 428-434, 1958.

IBGE - Instituto Brasileiro de Geografia e Estatística. Produção agrícola municipal SIDRA. Disponível em: <https://sidra.ibge.gov.br/tabela/5457>. Acesso em: 22 abr 2019.

NADIRI, A. A.; MOGHADDAM, A. A.; TSAI, F. T. C.; FIJANI, E. Hydrogeochemical analysis for Tasuj plain aquifer, Iran. Journal of Earth System Science, v. 122, p. 10911105, 2013. DOI: http://dx.doi.org/0.1007 /s12040-013-0329-4.

NEVES, A. L. R.; ALVES, M. P.; LACERDA, C. F.; GHEYI, H. R. Aspectos socioambientais e qualidade da água de dessalinizadores nas comunidades rurais de Pentecoste - CE. Revista Ambiente \& Água, v. 12, p. 124-135, 2017. DOI: http://dx.doi.org/10.4136/ambi-agua. 1722.

NÓBREGA, J. S.; SILVA, T. I. da; SILVA RIBEIRO, J. E da; VIEIRA, L. de S.; ANDRADE FIGUEIREDO, F. R.; FÁTIMA, R. T. de; ALCÂNTARA BRUNO, R. de L.; DIAS, T. J. Emergência e crescimento inicial de melancia submetida a salinidade e doses de ácido salicílico. Desafios - Revista Interdisciplinar da Universidade Federal do
Tocantins, v. 7, p. 162-171, 2020. DOI: http://dx.doi.org/10.20873/uftv7-8169.

PAGÈS, J.; HUDSON, F. Inter-laboratory comparison of sensory profiles: Methodology and results. Food Quality and Preference, v. 12, p. 297-309, 2001. DOI: http://dx.doi.org/10.1016/S0950-3293(01)00015-5.

$\mathrm{R}$ CORE TEAM. R: A language and environmental for statistical computing. Vienna: $\mathrm{R}$ Foundation for Statistical Computing, 2017. Disponível em: <https://cran.rproject.org/bin/windwos/base/ol d/3.4.3/>. Acesso em Jan. 2019.

RICHARDS, L. A. Diagnosis and improvement of saline and alkali soils. Washington: U. S. Department of Agriculture, 1954. 160p. (USDA, Agriculture Handbook, $60)$.

RODRIGUES, A. J. O.; NUNES, L. R. L.; NUNES, A. M. C.; UCHÔA, K. S. A. Efeito da adubação silicatada no cultivo de tomateiro sob estresse salino. Agropecuária Científica no Semiárido, v. 14, p. 141-148, 2018.

SAPORTA, G.; HATABIAN, G. Régions de confiance en analyse factorielle. In: DIDAY, E. 1. ed. Data analysis and informatics. Amsterdam: Elsevier, 1986. p. 499-508.

SARABI, B.; BOLANDNAZAR, S.; GHADERI, N.; TABATABAEI, S. J. Multivariate analysis as a tool for studying the effects of salinity in different melon landraces at germination stage. Notulae Botanicae Horti Agrobotanici Cluj-Napoca, v. 44, p. 264-271, 2016. DOI: http://dx.doi.org/10.15835/nbha44110234.

SILVA JÚNIOR, F. B.; SOUSA, G. G de.; SOUSA, J. T. M. de; LESSA, C. I. N.; SILVA, F. D. B da. Salt stress and ambience on the production of watermelon seedlings. Revista Caatinga, v. 33, p. 518-528, 2020. DOI: http://dx.doi.org/10.1590/1983-21252020v33n22rc. 
SOUSA, A. B. O. de; DUARTE, S. N.; SOUSA NETO, O. N. de; SOUZA, A. C. M.; SAMPAIO, P. R. F.; DIAS, C. T. dos S. Production and quality of mini watermelon cv. Smile irrigated with saline water. Revista Brasileira de Engenharia Agrícola e Ambiental, v. 20, p. 897-902, 2016. DOI: http://dx.doi.org/10.1590/18071929/agriambiv 20n10p897-902.
VIANA, P. C.; FREITAS, F. T. O. de; SILVA, N. D. da; SOARES, T. M.; PAZ, M. G. F. da. Estatística multivariada como ferramenta descritiva na análise sensorial de alface hidropônica produzida com águas salobras. Revista Brasileira de Agricultura Irrigada, v. 12, p. 27252730 , 2018.

DOI: http://dx.doi.org/10.7127/RBAI.V12N400959. 